\title{
Reconocimiento del efecto de Cinara cupressi (Hemiptera: Aphididae) en el estado sanitario de Austrocedrus chilensis mediante imágenes multiespectrales
}

\author{
Recognition of the effect of Cinara cupressi (Hemiptera: Aphididae) in the sanitary condition of \\ Austrocedrus chilensis by multispectral images
}

\author{
Marco A Peña ${ }^{a *}$, Scott H Altmann ${ }^{a}$ \\ *Autor de correspondencia: aUniversidad Mayor, Centro de Estudios de Recuros Naturales, Oterra, Camino La Pirámide 5750, \\ Huechuraba, Santiago, Chile, tel.: 56-2-3281430, marco.pena@umayor.cl
}

\begin{abstract}
SUMMARY
This work examined the utility of multispectral remote sensing to recognize the effects produced by infestation of the cypress aphid (Cinara cupressi) in the sanitary condition of the cordillera cypress (Austrocedrus chilensis). The study area is Fundo Los Cipreses, located in the foothills sector of the San Fernando County, Maule Region of Chile. Procedures focused on the recognition of variations in foliage vigor and turgidity of infested A. chilensis using ASTER images acquired at periods of preinfestation (March, 2003) and infestation (March, 2008). Both parameters were measured by the normalized difference vegetation index (NDVI) and the normalized difference water index (NDWI), respectively, for a set of geographic points corresponding to two main damage categories detected in the field (moderate and severe). In the study period a clear decrease in the NDVI and NDWI of the severely damaged individuals was evidenced, while those individuals that present a moderate damage did not show important differences for both indices. Results suggest that multispectral images are useful to detect severe damage in a stand. In the future it is expected that further development of the capacities of this type of product, complemented with hyperspectral images, allow for effective monitoring of $C$. cupressi infestation in other areas of the country.
\end{abstract}

Key words: vegetation indices, vegetation health status, Austrocedrus chilensis, Cinara cupressi, multispectral imagery.

\section{RESUMEN}

Este trabajo examinó la utilidad de la observación remota multiespectral para reconocer los efectos producidos por la infestación del pulgón del ciprés (Cinara cupressi) en el estado sanitario de las formaciones de ciprés de la cordillera (Austrocedrus chilensis) del Fundo Los Cipreses, localizado en el sector precordillerano de la comuna de San Fernando, VI Región de Chile. Los procedimientos se centraron en reconocer variaciones de verdor y turgencia en el follaje de A. chilensis infestados usando imágenes ASTER adquiridas en los períodos de preinfestación (marzo de 2003) e infestación (marzo de 2008). Ambos parámetros fueron medidos mediante el índice de vegetación de diferencia normalizada (NDVI) y el índice de agua de diferencia normalizada (NDWI), respectivamente, para una serie de puntos geográficos correspondientes a dos principales categorías de daño constatadas en terreno (moderado y severo). En el período de estudio se evidenció una clara disminución de NDVI y NDWI en los individuos severamente dañados, mientras que aquellos individuos que presentan un daño moderado no mostraron diferencias importantes para ambos índices. Los resultados sugieren que las imágenes multiespectrales son útiles para reconocer daño de infestación severo en un rodal. A futuro se espera que un mayor desarrollo de las capacidades de este tipo de producto, complementado con imágenes hiperespectrales, permita efectivamente monitorear la infestación de $C$. cupressi en el país.

Palabras clave: índices de vegetación, estado sanitario, Austrocedrus chilensis, Cinara cupressi, imágenes multiespectrales.

\section{INTRODUCCIÓN}

La observación remota o teledetección es la disciplina que deriva información de los elementos de la superficie terrestre mediante la detección y análisis de la energía radiada por ellos. Mediante la generación de imágenes multiespectrales ésta ha proporcionado datos estrechamente relacionados con varios atributos biofísicos y bioquímicos de la vegetación, permitiendo interpolar y extrapolar mediciones de terreno sobre áreas extensas e inaccesibles
(Iverson et al. 1989, Wulder y Franklin 2003). Este tipo de imágenes ha permitido caracterizar el estado sanitario de la vegetación a través del análisis de las señales que diversos de sus componentes (pigmentos, agua) producen en porciones específicas del espectro electromagnético (bandas espectrales).

Específicamente, en las longitudes de onda del rojo (R) la clorofila foliar absorbe fuertemente la radiación solar incidente, alcanzando un máximo alrededor de los $0,7 \mu \mathrm{m}$. En las longitudes de onda del infrarrojo cercano (IC) la 
estructura interna foliar refleja fuertemente la radiación solar incidente, alcanzando un máximo entre los 0,8 y $0,9 \mu \mathrm{m}$. En las longitudes de onda del infrarrojo de onda corta (IOC) el agua foliar absorbe fuertemente la radiación solar incidente, alcanzando máximos alrededor de los 1,4, 1,9 y $2,7 \mu \mathrm{m}$ (Lillesand et al. 2004, Aronoff 2005). Es mediante la combinación aritmética de estas bandas que ha sido posible derivar índices de verdor y contenido de agua del vegetal desde imágenes multiespectrales. Así, mientras mayor es la diferencia entre los valores de reflectancia (proporción de radiación solar incidente que es reflejada por un elemento) registrados en las bandas $\mathrm{R} e$ IC para un píxel dado de la imagen (elemento mínimo de información contenida en una imagen), mayor es la proporción de follaje verde y vigoroso contenido en él. Del mismo modo, mientras mayor es la diferencia entre los valores de reflectancia registrados en las bandas IC e IOC para un píxel dado de la imagen, mayor es la proporción de follaje turgente contenido en él (Hunt y Rock 1989, Lillesand et al. 2004, Lucas et al. 2004).

A pesar de los numerosos índices de verdor de vegetación que han sido introducidos en la literatura, la simpleza, relativa robustez y versatilidad del índice de vegetación de diferencia normalizada (normalized difference vegetation index, NDVI) ha hecho de éste el más profusamente empleado para caracterizar el estado sanitario de las coberturas vegetales, existiendo muchos estudios que han probado su relación con mediciones en terreno de abundancia vegetal, índice de área foliar y biomasa, entre otros parámetros (Karnieli et al. 2001, Gitelson et al. 2002, Asner et al. 2003, Baret y Buis 2008). Dentro de sus capacidades está la de minimizar los efectos causados por sombras topográficas (pues al basarse en la división de bandas arroja una razón similar entre la vegetación ubicada en ladera sombreada e iluminada) y por errores de calibración del sensor (debido a la normalización del índice), así como la de expresar los valores en una escala que va desde -1 a 1 , donde los valores inferiores o iguales a 0 corresponden a suelo desnudo y aquellos superiores corresponden a suelo vegetado, representando el valor 1 a un píxel saturado de vegetación verde (Eastman 2006, Zhang et al. 2006).

Debido a lo anterior, el NDVI ha sido usado para rastrear y evaluar los daños ocasionados por infestaciones de insectos en bosques, usualmente caracterizados por la defoliación y disminución del contenido de pigmentos de clorofila foliar (i.e. clorosis). Las aproximaciones metodológicas empleadas se han basado en estimar las diferencias que este índice presenta entre áreas infestadas y no infestadas (Carter et al. 1998), entre períodos de preinfestación e infestación (Young y Morton 2002) o entre períodos de baja y alta infestación (Komura et al. 2003). Por otra parte, otros trabajos han estimado las diferencias que presentan separadamente las reflectancias de las bandas R e IC entre áreas infestadas y no infestadas (Vogelmann y Rock 1989, Ekstrand 1990, Kharuk et al. 2003).
Hay una proporción relativamente menor de trabajos que han aplicado índices de contenido de agua para estudiar infestaciones de insectos en bosques (Huete y Didan 2004). No obstante, su consideración es importante, pues aunque ha sido demostrada una correspondencia entre turgencia y verdor vegetal (Stimson et al. 2005), esta relación no siempre ocurre y debe ser considerada caso a caso (Niemann y Visintini 2004). Así, por ejemplo, aunque Vogelmann (1990) encontró una fuerte correlación entre el NDVI y el índice de contenido de agua de razón simple ( $\mathrm{r} \approx 1)$, así como una relación significativa $(P<0,001)$ entre ambos índices y la defoliación de bosques infestados, concluyó que el índice de contenido de agua fue más apropiado para diferenciar categorías de daño moderado y severo.

El índice de agua de diferencia normalizada (normalized difference water index, NDWI) ha sido usado en varios estudios forestales (Stimson et al. 2005, Ito et al. 2007). Posee las mismas propiedades que el NDVI, incluyendo la escala de valores; en que 1 representa a un píxel saturado de agua. Así, mediante el empleo de ambos índices es posible caracterizar el estado sanitario del vegetal y realizar comparaciones temporales entre valores debidamente sujetos a correcciones radiométricas.

En Chile, en agosto de 2003, se detectó la presencia de Cinara cupressi (Buckton) (Hemiptera: Aphididae) agg., un áfido caracterizado por una gran capacidad de establecerse en áreas ajenas a su rango natural de distribución y considerado como una de las cien especies invasoras más dañinas del mundo (Lowe et al. 2000). Actualmente, C. cupressi se encuentra distribuido por todo el país, atacando especies nativas y exóticas de la familia Cupressaceae, especialmente al ciprés de la cordillera (Austrocedrus chilensis (D. Don) Pic. Serm. et Bizzarri), sobre el que causa decoloración, defoliación y muerte, tanto por depredación como por efectos secundarios. El ataque del insecto suele acentuarse durante el período estival, debido a que las altas temperaturas favorecen su desarrollo y reproducción (Aguayo et al. 2005, Holmgren 2006). En 2005, un estudio realizado a nivel nacional detectó la presencia de $C$. cupressi en 92\% de los puntos de muestreo para A. chilensis con presencia de Cinara spp., distribuidos desde la Región de Tarapacá hasta la Región de Aisén (INFOR 2008). Otra causa de declinación de bosques de A. chilensis ha sido atribuida a la pudrición de sus raíces por hongos patogénicos (Phytophthora austrocedrae Gresl. et EM Hansen, sp. nov.) (Greslebin et al. 2005, Greslebin et al. 2007), un fenómeno que ha recibido atención especial en Argentina y que ha sido vinculado al "mal de ciprés". Por otra parte, la incidencia de factores abióticos también ha sido atribuida como una causa de disminución de vigor de estos bosques (Baccalá et al. 1998).

Austrocedrus chilensis es una conífera nativa de Chile y Argentina, considerada vulnerable a la extinción según los criterios de la Unión Internacional para la Conservación de la Naturaleza y de los Recursos Naturales (UICN 2001). 
Se extiende por más de $1.200 \mathrm{~km}$ a lo largo de las vertientes occidentales y orientales de la cordillera central de Los Andes (Rodríguez 2004). En Chile se distribuye en forma de parches aislados desde los $32^{\circ} 39^{\prime}$ S (Región de Valparaíso) hasta los $44^{\circ} \mathrm{S}$ (Región de Los Lagos), concentrándose mayoritariamente en la cordillera andina (Schmithüsen 1960, Schlegel 1962, Donoso 1982, Rodríguez 2004). De acuerdo a Donoso (1981) las formaciones de esta especie pertenecen a la comunidad "tipo forestal de ciprés de la cordillera". Desde una perspectiva bioclimática estas formaciones pertenecen a la clasificación "bosque caducifolio mediterráneo andino" de Nothofagus obliqua (Mirb.) Oerst. y A. chilensis (Luebert y Pliscoff 2006). La especie habita terrenos que fluctúan entre los 400 y 1.800 m s.n.m., de distinta composición (rocosos, volcánicos y aluviales) y con pendiente y exposición variables. Los rodales varían en su densidad y estructura de edad, y generalmente se asocian a un sotobosque esclerófilo (Lithraea caustica (Mol.) Hook et Arn.) y a Nothofagus glauca (Phil.) Krasser (Donoso 1982, Donoso 1993).

El presente trabajo explora las capacidades que ofrece la observación remota de tipo multiespectral para reconocer los efectos que produce la infestación de $C$. cupressi en el estado sanitario de las formaciones de A. chilensis ubicadas en el fundo Los Cipreses, perteneciente a la localidad de Las Peñas, comuna de San Fernando, Región del Maule, Chile, donde una actividad significativa del áfido ha sido reportada a partir de septiembre de 2004 (Holmgren 2006, Baldini et al. 2008, INFOR 2008). Para ello, los parámetros de NDVI (asociado al vigor del follaje) y NDWI (asociado a turgencia del follaje) fueron obtenidos desde imágenes satelitales ASTER (Advanced Spaceborne Thermal Emission and Reflection Radiometer) adquiridas antes de la infestación (marzo de 2003) y durante la infestación (marzo de 2008), y fueron comparados y analizados para una serie de puntos geográficos de A. chilensis correspondientes a las dos principales categorías de daño constatadas en terreno (moderado y severo).

Aunque en la actualidad existen numerosos tipos de imágenes multiespectrales en el mercado, sólo las imágenes ASTER cumplen con la resolución espacial, espectral y temporal requerida para este tipo de estudio. Así, por ejemplo, las imágenes multiespectrales de alta resolución espacial (Ikonos, Quickbird, Orbview, Geoeye, Komposat y Formosat) no cuentan con bandas IOC (impidiendo el cálculo del NDWI). Por otro lado, aunque una imagen Landsat cuenta con bandas IOC, ésta tiene un mayor tamaño de píxel en las bandas visible e IC que las imágenes ASTER (30 m versus $15 \mathrm{~m}$ ) (degradando el nivel de detalle espacial de la superficie retratada). Finalmente, aunque las imágenes ATLAS (Airborne Terrestrial Applications Sensor) cuentan con bandas de alta resolución espacial en las bandas visible, IC e IOC, su cobertura mundial es muy baja (no existen imágenes de archivo para el área de estudio).

\section{MÉTODOS}

Área de estudio. El predio se localiza en la cuenca del río Tinguiririca, en la precordillera andina de la Región del Maule, Chile, aproximadamente entre las coordenadas $34^{\circ}$ $46^{\prime} \mathrm{S}$ y $40^{\circ} 47^{\prime} \mathrm{O}$. El área posee un relieve montañoso que cubre una superficie alrededor de 2.000 ha, con una altura promedio cercana a $900 \mathrm{~m}$ s.n.m. y se inserta en un clima mediterráneo frío. Los bosques de A. chilensis conforman renovales multietáneos que se distribuyen heterogéneamente formando agrupaciones en algunos sectores favorables para su crecimiento (laderas umbrías), donde ocupan la posición dominante del dosel superior, mezclándose en algunos sectores de ladera con pequeños rodales de Nothofagus spp. A menor altura estos bosques limitan con formaciones de bosque esclerófilo y terrenos agrícolas, distribuidos en el fondo del valle. A mayor altura limitan con suelos rocosos donde la vegetación prevaleciente corresponde a pastos, arbustos y árboles achaparrados. En septiembre del año 2004 fue detectada la presencia de $C$. cupressi y en la actualidad todos los rodales de A. chilensis exhiben daño inducido por el áfido, que se manifiesta en la presencia de follaje parcialmente clorótico y necrótico. La figura 1 presenta una visión tridimensional del territorio en que se localiza el área de estudio (delimitada por el óvalo).

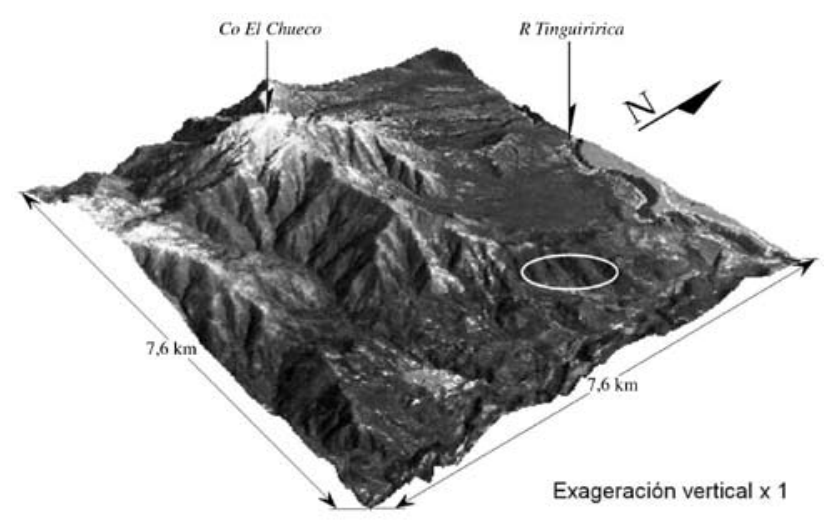

Figura 1. Representación tridimensional del relieve del área de estudio y sus inmediaciones. El óvalo delimita los rodales en que se efectuaron las mediciones de terreno.

Three-dimensional representation of the relief of the study area and its surroundings. The oval delimits the stands in which field measurements were made.

Imágenes satelitales. Este trabajo empleó dos imágenes satelitales ASTER adquiridas el 1 de marzo de 2003 (período de preinfestación) y el 30 de marzo de 2008 (período de infestación), a fin de analizar la vegetación de interés en similares condiciones fenológicas (alto estado de vigorosidad) y durante el período de mayor actividad y reproducción del áfido (verano). Este tipo de imágenes se compone de nueve bandas de energía reflejada: dos en la región visible, una en la región IC y seis en la región 
IOC. La resolución espacial es de $15 \mathrm{~m}$ en las bandas visible e IC, y $30 \mathrm{~m}$ en las bandas IOC.

Preprocesamiento radiométrico y geométrico de las imágenes. Los niveles digitales de las bandas de ambas imágenes ASTER fueron convertidos a radiancias calibradas empleando las fórmulas de conversión provistas por el Land Processes Distributed Active Data Center y los parámetros de calibración radiométrica contenidos en los archivos auxiliares de cada imagen. Luego, ambas imágenes fueron georreferenciadas mediante la aplicación de un método de triangulación usando un remuestreo de vecino más cercano con resolución espacial de $15 \mathrm{~m}$. Para ello, a cada imagen se le aplicaron automáticamente los parámetros de órbita satelital y el modelo geométrico contenidos en su archivo auxiliar, usando el programa ENVI versión 4.5. Los parámetros de proyección geográfica asignados a las imágenes fueron: coordenadas Universal Transversal de Mercator (UTM), datum Sistema Geodésico Mundial 1984 (WGS84), huso 19 Sur. El error posicional de las imágenes georreferenciadas mediante este procedimiento de corrección geométrica fue inferior al valor mínimo aceptable (i.e. error medio de la raíz cuadrada < medio píxel).

Para suprimir la radiancia extraña añadida a los píxeles de las imágenes, debido al esparcimiento de radiación que producen los aerosoles atmosféricos (i.e. efecto de niebla) y las superficies aledañas a cada píxel (i.e. efecto de adyacencia), fue aplicado el modelo de corrección atmosférica FLAASH (Fast Line-of-Sight Atmospheric Analysis of Spectral Hypercubes) disponible en el programa ENVI versión 4.5, que se basa en cálculos MODTRAN (Moderate Resolution Atmospheric Transmission) para modelar las condiciones atmosféricas imperantes al momento de paso de la plataforma por un área dada. Para su operación requiere la hora y la fecha de adquisición de la imagen, la altitud del satélite, la altitud promedio del relieve retratado en la imagen y la coordenada central de la escena, además de un conjunto de parámetros asociados al modelamiento de la atmósfera del área de interés. El resultado de este procedimiento fueron imágenes en unidades de reflectancia superficial aparente (aquella que incluye el efecto de la atmósfera en el flujo radiante descendente y excluye el efecto de la atmósfera en el flujo radiante ascendente) escaladas en valores comprendidos entre 0 (valor equivalente a un cuerpo negro o emisor perfecto) y 10.000 (valor equivalente a un cuerpo blanco o reflector perfecto).

Cálculo de índices de vegetación. El NDVI fue estimado mediante la siguiente combinación aritmética de bandas [1]:

$$
\mathrm{NDVI}=\mathrm{IC}-\mathrm{R} / \mathrm{IC}+\mathrm{R}
$$

Donde:

IC: banda de la región del infrarrojo cercano (banda 3).

$\mathrm{R}$ : banda de la región del rojo (banda 2).
La fórmula del NDWI es idéntica a la del NDVI, sólo que la banda $\mathrm{R}$ es reemplazada por la banda IOC (banda 4) (Ito et al. 2007).

Colección de datos en terreno. Para reconocer variaciones en el estado sanitario de A. chilensis infestados por $C$. $\mathrm{cu}$ pressi, fueron colectados con un GPS (global positioning system) puntos correspondientes a las dos categorías de daño predominantes dentro del área de estudio: moderado y severo, para luego superponerlos a cada imagen y extraer sus respectivos valores de NDVI y NDWI. Todos los puntos fueron colectados con una precisión posicional de $\pm 5 \mathrm{~m}$. Nueve puntos fueron muestreados en rodales correspondientes a la categoría de daño moderado y cuatro puntos fueron muestreados en rodales correspondientes a la categoría de daño severo. Las observaciones realizadas en dos visitas a terreno a fines del año 2007 permitieron constatar que prácticamente todas las formaciones de $A$. chilensis del área de estudio se encuentran infestadas por C. cupressi, exhibiendo predominantemente una categoría de daño moderado.

La categoría de daño moderado representa a rodales de A. chilensis en que abundan individuos con manifestaciones de clorosis y necrosis en su follaje y con un aspecto general de baja vigorosidad, que se evidencia por la decoloración y pérdida de biomasa del follaje. La categoría de daño severo representa a rodales de $A$. chilensis cuyos individuos presentan una agudización y generalización de los síntomas anteriormente mencionados, con ramas muertas e incluso ejemplares muertos o al borde de la muerte.

Con el fin de minimizar las fuentes de distorsión de los índices calculados para los puntos de ambas categorías de daño, las áreas en que estos últimos fueron colectados debieron cumplir con los siguientes requisitos: 1) Baja presencia de suelo desnudo: con el objeto de disminuir la alteración que puede producir el suelo en la reflectancia proveniente del objeto de interés, en este caso el follaje de A. chilensis, se privilegió la colección de puntos en áreas con la menor proporción de suelo desnudo posible. 2) Cobertura vegetal pura: con el objeto de obtener reflectancias provenientes predominantemente del objeto de interés, se privilegió la colección de puntos en áreas con un dosel superior compuesto principal o exclusivamente por A. chilensis. 3) Superficie mínima homogénea: con el propósito de asegurar que los puntos muestreados en terreno correspondieran espacialmente con sus respectivos valores de índices de vegetación calculados desde cada imagen, cada uno de ellos fue colectado en el centro de áreas pertenecientes a una misma categoría de daño y con una superficie superior a $900 \mathrm{~m}^{2}$ (cuatro píxeles de la imagen). Pocas áreas severamente dañadas cumplieron con este criterio, razón que explica el bajo tamaño de la muestra para esa categoría. En cambio, las áreas moderadamente dañadas presentaron extensiones superiores a $8.000 \mathrm{~m}^{2}$. 
Para la comparación de valores de NDVI y NDWI se ocuparon las pruebas no paramétricas Mann Whitney U y Kolmogorov-Smirnov, debido a que los datos no presentaron una distribución normal.

\section{RESULTADOS}

Los valores de NDVI y NDWI promedio de los puntos colectados en cada categoría de daño según las fechas de estudio son presentados en el cuadro 1. Entre los períodos de preinfestación e infestación el NDVI de la categoría de daño moderado presenta una sutil disminución $(\Delta=0,02)$, mientras que el NDVI de la categoría de daño severo experimenta una disminución más apreciable $(\Delta=0,13)$. De forma similar, entre los períodos de preinfestación e infestación el NDWI de la categoría de daño moderado presenta una sutil variación $(\Delta=0,01)$, mientras que el NDWI de la categoría de daño severo experimenta una disminución más apreciable $(\Delta=0,07)$.

Las pruebas estadísticas anteriormente mencionadas permitieron determinar si entre las dos fechas de estudio hubo una diferencia significativa en los valores de NDVI y de NDWI de las categorías de daño moderado y severo, respectivamente. Para la primera categoría los resultados fueron: NDVI, valor $\mathrm{Z}=0,5309, P=0,60$ (prueba Mann-Whitney U); NDWI, valor Dmn $=0,3333, P=0,73$ (prueba Kolmogorov-Smirnov). Para la segunda categoría los resultados fueron: NDVI, valor $\mathrm{Z}=2,0207, P=0,04$ (prueba Mann-Whitney U); NDWI, valor Dmm = 0,7500, $P=0,23$ (prueba Kolmogorov-Smirnov).

Cuadro 1. NDVI y NDWI promedios de cada categoría de daño según fecha de adquisición de la imagen $( \pm \mathrm{EE})$.

Mean NDVI and NDWI of each damage category according to the image acquisition date $( \pm \mathrm{SE})$.

\begin{tabular}{cccc}
\hline \multirow{2}{*}{ Promedio } & \multirow{2}{*}{$\begin{array}{c}\text { Categoría } \\
\text { de daño }\end{array}$} & \multicolumn{2}{c}{ Imagen } \\
\cline { 3 - 4 } & Marzo 2003 & Marzo 2008 \\
\hline \multirow{2}{*}{ NDVI } & Moderado & $0,58(0,027)$ & $0,56(0,026)$ \\
& Severo & $0,55(0,040)$ & $0,42(0,032)$ \\
\hline \multirow{2}{*}{ NDWI } & Moderado & $0,14(0,032)$ & $0,15(0,029)$ \\
& Severo & $0,17(0,028)$ & $0,1(0,067)$ \\
\hline
\end{tabular}

El cuadro 2 permite apreciar que para ambas fechas de estudio las reflectancias de las bandas $\mathrm{R}$ e IOC de la categoría de daño moderado son menores que aquellas correspondientes a la categoría de daño severo. En 2003, la reflectancia de la banda del IC de la categoría de daño moderado es menor que aquella correspondiente a la categoría de daño severo. En 2008, la reflectancia de la banda del IC de la categoría de daño moderado es mayor que aquella correspondiente a la categoría de daño severo.

Para la categoría de daño moderado no hubo diferencias significativas entre fechas de estudio para las reflectancias de la banda $\mathrm{R}$ (valor $\mathrm{Z}=0,5746, P=0,57$, prueba Mann-Whitney U), IC (valor $\mathrm{Z}=0,9272, P=0,39$, prueba Mann-Whitney U) e IOC (valor Dmm =0,2222, $P=0,99$, prueba Kolmogorov-Smirnov). Para la categoría de daño severo hubo una diferencia entre fechas de estudio para la reflectancia de la banda IC (valor Dmm $=2,3094$, $P=0,02$, prueba Mann-Whitney $\mathrm{U})$, pero no así para las bandas $\mathrm{R}$ (valor $\mathrm{Dmm}=0,5000, P=0,77$, prueba Kolmogorov-Smirnov) e IOC (valor $\mathrm{Z}=1,1832, P=0,24$, prueba Mann-Whitney U).

Las curvas de valores de reflectancia de cada categoría de daño según fecha de estudio son presentadas en la figura 2. La curva de la categoría de daño moderado de 2008 presenta un menor máximo de reflectancia en las longitudes de onda del IC $(0,8 \mu \mathrm{m})$ que aquella correspondiente a 2003, manteniendo esa diferencia de reflectancia hacia las longitudes de onda del IOC. La curva de reflectancia de la categoría de daño severo de 2008 presenta un mayor máximo de reflectancia en las longitudes de onda del $\mathrm{R}(0,7 \mu \mathrm{m})$ y el IOC $(2,3 \mu \mathrm{m})$, y un menor máximo de reflectancia en las longitudes de onda del IC $(0,8 \mu \mathrm{m})$, que aquella correspondiente a 2003.

\section{DISCUSIÓN Y CONCLUSIONES}

Este trabajo exploró las capacidades que ofrece la observación remota de tipo multiespectral para reconocer los efectos que produce la infestación de $C$. cupressi en el estado sanitario de las formaciones de A. chilensis. Para ello fueron empleadas imágenes adquiridas en los períodos de preinfestación e infestación de fechas similares

Cuadro 2. Reflectancia promedio de cada categoría de daño para las bandas rojo (R), infrarrojo cercano (IC) e infrarrojo de onda corta (IOC) de cada imagen $( \pm \mathrm{EE})$.

Mean reflectance of each damage category for the red (R), near-infrared (NIR) and shortwave infrared (SWIR) bands of each image ( \pm SE).

\begin{tabular}{|c|c|c|c|c|c|c|}
\hline \multirow{3}{*}{$\begin{array}{c}\text { Categoría de } \\
\text { daño }\end{array}$} & \multicolumn{6}{|c|}{ Imagen } \\
\hline & \multicolumn{3}{|c|}{ Marzo 2003} & \multicolumn{3}{|c|}{ Marzo 2008} \\
\hline & $\mathrm{R}$ & IC & IOC & $\mathrm{R}$ & IC & IOC \\
\hline Mediano & $533,22(43,9)$ & $2.030,44(79,5)$ & $1.520,00(101,9)$ & $540,22(53,7)$ & $1,943,22(98,0)$ & $1.438,55(69,9)$ \\
\hline Severo & $657,25(92,2)$ & $2.270,00(130,5)$ & $1.606,25(31,8)$ & $735,00(33,3)$ & $1,819,50(59,0)$ & $1.625,75(162,1)$ \\
\hline
\end{tabular}



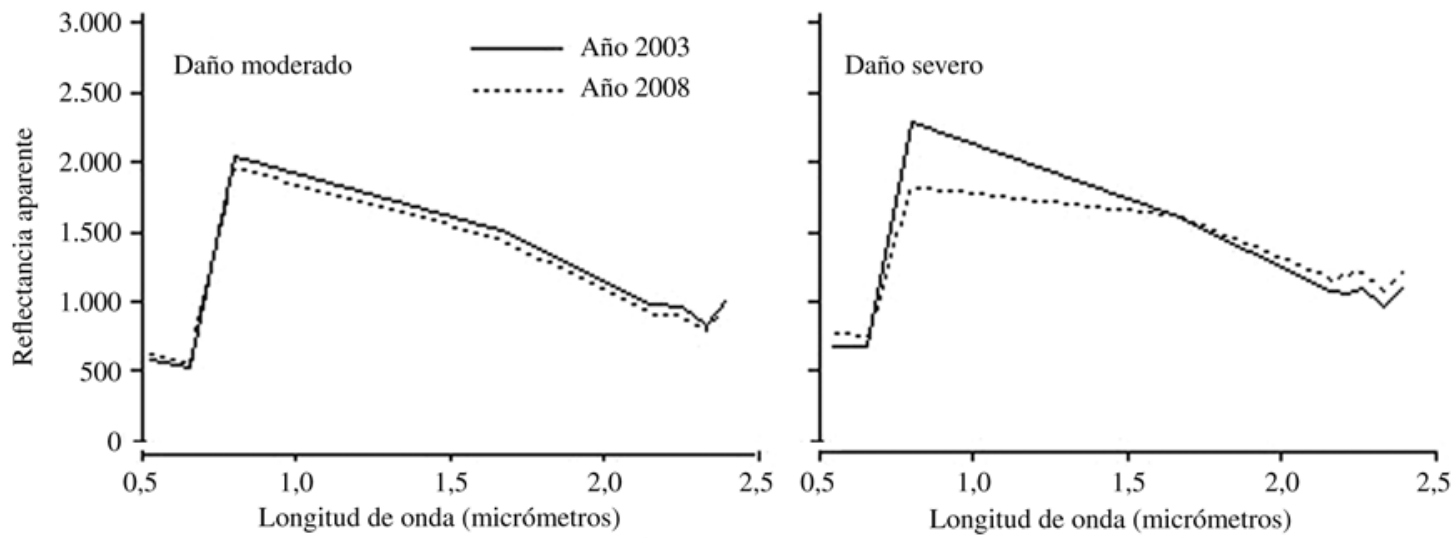

Figura 2. Curvas de reflectancia espectral promedio de los puntos muestreados para las categorías de daño moderado y severo.

Mean spectral reflectance curves of the points sampled for the moderate and severe damage categories.

(fechas de aniversario), con el objeto de minimizar la influencia de la variabilidad fenológica de A. chilensis en los análisis. Esta aproximación metodológica ha sido previamente empleada en estudios de infestaciones de bosques (Vogelmann y Rock 1989, Vogelmann 1990, Young y Morton 2002, Kharuk et al. 2003). Por otra parte, este trabajo levantó datos de terreno durante la misma estación en que fue adquirida la imagen del período de infestación (verano 2007-2008), de manera que los datos de terreno y remotos de $A$. chilensis correspondan a una similar condición fisiológica.

Los resultados sugieren que los efectos que produce la infestación del áfido sobre el estado sanitario de los rodales de A. chilensis estudiados son particularmente reconocibles en la categoría de daño severo. Tanto el NDVI como el NDWI de esta categoría experimentaron una notoria disminución entre los períodos de preinfestación e infestación. En cambio, el NDVI y NDWI de la categoría de daño moderado se mantuvieron muy similares entre ambos períodos. Para la categoría de daño severo, el aumento de la reflectancia en las longitudes de onda del $\mathrm{R}$ en el año 2008 revela que estos rodales experimentaron una disminución de pigmentos de clorofila en su follaje, mientras que la disminución de la reflectancia en las longitudes de onda del IC en el año 2008 revela que estos rodales experimentaron una disminución de follaje verde. Por último, el aumento de la reflectancia en las longitudes de onda del IOC entre ambos períodos indica que estos rodales experimentaron una disminución de follaje turgente. Es posible que un mayor tamaño muestral de la categoría de daño severo hubiera revelado resultados significativos entre fechas para los valores de NDWI y las bandas IOC y R. Para la categoría de daño moderado en tanto, la disminución de la reflectancia de las longitudes de onda IC entre ambos períodos indica que estos rodales experimentaron una disminución de follaje verde, aunque menos apreciable que en el caso de la categoría de daño severo.
En el período de preinfestación, destaca la pequeña diferencia que presenta el NDVI de la categoría de daño severo en relación con aquel de la categoría de daño moderado, lo que sugiere que en 2003 los rodales pertenecientes a ambas categorías de daño debieron presentar un similar estado sanitario. En el período de infestación los valores de NDVI de ambas categorías de daño presentan una diferencia mucho mayor, lo que remarca los efectos de la infestación de $C$. cupressi en el verdor de la categoría de daño severo.

Aunque los valores de NDVI varían de un trabajo a otro en función de múltiples factores como: tipo de sensor, en especial su resolución radiométrica y ancho de bandas; procedimientos de corrección atmosférica y calibración radiométrica aplicados a la imagen; condiciones de iluminación al momento de paso del satélite, y estado fenológico y fisiológico de las diferentes especies, entre otros, usualmente valores cercanos o superiores a 0,8 corresponden a áreas con una alta abundancia de follaje, mientras que valores cercanos o inferiores a 0,3 corresponden a una cobertura de vegetación espaciada o poco vigorosa (Derek et al. 2003). Estos valores de referencia permiten apreciar que los puntos de A. chilensis muestreados antes y durante la infestación poseen una moderada cobertura de follaje.

En el fundo Los Cipreses, Baldini et al. (2008) observaron la presencia de ramas muertas en todos los individuos de A. chilensis muestreados $(\mathrm{n}=100)$ y defoliación y clorosis en más de un $70 \%$ de ellos. El INFOR (2008) en tanto, encontró que el $42 \%$ de los individuos de A. chilensis muestreados $(\mathrm{n}=3.230)$ en 2005 y 2008 , presentaron una pérdida de follaje y ramas secas superior a $50 \%$. Estos resultados dan cuenta de la generalización de la infestación presente en el área de estudio, la que además se caracteriza por un daño homogéneo. Muchos estudios vinculados con la detección remota multiespectral de daños causados por infestaciones de insectos en bosques han presentado dificultades para diferenciar con 
claridad categorías de daño, principalmente debido a la insuficiencia que poseen los datos multiespectrales para proveer señales espectrales distintivas asociadas a niveles de defoliación (Radeloff et al. 1999). A este respecto, las pequeñas diferencias de NDVI obtenidas en este trabajo para la categoría de daño moderado permiten suponer que el uso de este tipo de imágenes presenta una similar limitante para rastrear daños en A. chilensis infestados por C. cupressi.

Aunque el NDVI y el NDWI proveen información relevante sobre el estado sanitario del vegetal, cabe destacar que tecnologías de observación remota relativamente nuevas (i.e. hiperespectral) podrían ser de mayor utilidad para rastrear los daños causados por el áfido a lo largo del país. A este respecto, numerosos trabajos han probado la utilidad que presentan las imágenes hiperespectrales (capaces de detectar datos en cientos de bandas a lo largo del espectro visible, IC e IOC) para identificar y caracterizar en detalle bosques infestados por insectos (Coops et al. 2003, Lawrence y Labus 2003, Entcheva Campbell et al. 2004, Stone y Coops 2004). Ello se debe a la gran capacidad que éstas poseen para diferenciar el verdor en áreas sobre las que usualmente los datos multiespectrales se saturan (debido a que miden energía en bandas muy anchas), y para proveer de muchos otros parámetros fisiológicos del vegetal, asociados a la presencia de pigmentos como antocianinas, carotenoides, xantofilas, o nutrientes como nitrógeno, fósforo y potasio. Las señales que estos componentes producen en el rango espectral óptico no son detectables mediante datos multiespectrales, debido a que miden reflectancias a lo largo de bandas demasiado anchas (Treitz y Howarth 1999, Lucas et al. 2004, Blackburn 2007, Govender et al. 2007).

En estudios relacionados con infestaciones en bosques, las imágenes ASTER han sido mucho menos aplicadas que las imágenes multiespectrales (Landsat), probablemente debido a que las primeras son más nuevas que las segundas (el sensor ASTER fue puesto en órbita en diciembre de 1999 y las misiones Landsat datan de julio de 1972). Asimismo, el NDWI ha sido mucho menos aplicado que el NDVI, debido a que este último tiene la propiedad de dar cuenta simultáneamente de dos atributos de la vegetación fuertemente sensibles a los efectos de cualquier agente estresor, además de estar ampliamente validado en estudios de diversa naturaleza.

Los resultados obtenidos recientemente por Peña y Altmann (2009) soportan el uso de índices de vegetación derivados desde imágenes hiperespectrales para reconocer y caracterizar con mayor detalle los efectos que produce el ataque de $C$. cupressi sobre la fisiología de A. chilensis. De esta forma, durante los próximos años se espera profundizar en la capacidad de las imágenes multiespectrales para monitorear y controlar oportuna y selectivamente esta plaga a través de su complemento con información derivada desde imágenes hiperespectrales.

\section{REFERENCIAS}

Aguayo J, A Sartori, A Baldini. 2005. El complejo Cinara cupressi (Hemiptera: Aphididae) una amenaza para las cupresáceas nativas de Chile. Nota Técnica 23, № 46. Santiago, Chile. Programa de Protección Fitosanitaria Forestal, Corporación Nacional Forestal. 7 p.

Aronoff S. 2005. Remote sensing for GIS managers. New York, USA. ESRI Press. 487 p.

Asner G, J Hicke, D Lobell. 2003. Per-pixel Analysis of forest structure: Vegetation indices, spectral mixture analysis and canopy reflectance modeling. In Wulder MA, SE Franklin eds. Remote Sensing of Forest Environments: Concepts and Case Studies. Dordrecht, Holland. Kluwer Academic Publishers. p. 209-254.

Baccalá N, P Rosso, M Havrylenko. 1998. Austrocedrus chilensis mortality in the Nahuel Huapi National Park (Argentina). Forest Ecology and Management 109: 261-269.

Baldini A, J Oltremari, A Holmgren. 2008. Efecto de Cinara cupressi (Hemiptera: Aphididae) sobre el ciprés de la cordillera (Austrocedrus chilensis) después de aplicar control químico. Ciencia e Investigación Agraria 35 (3): 341-250.

Baret F, S Buis. 2008. Estimating canopy characteristics from remote sensing observations: review of methods and associated problems. In Liang S ed. Advances in Land Remote Sensing. New York, USA. Springer. p. 173-201.

Blackburn GA. 2007. Hyperspectral remote sensing of plant pigments. Journal of Experimental Botany 58 (4): 855-867.

Carter GA, RS Michael, T Haley. 1998. Airborne detection of southern pine beetle damage using key spectral bands. Canadian Journal of Forest Research 28: 1040-1045.

Coops N, M Standford, K Old, M Dudzinski, D Culvenor, C Stone. 2003. Assessment of Dothistroma Needle Blight of Pinus radiata using airborne Hyperspectral imagery. Phytopathology 93 (12): 1524-1532.

Derek RP, PM Teillet, MA Wulder. 2003. Radiometric image processing. In Wulder MA, SE Franklin eds. Remote Sensing of Forest Environments: Concepts and Case Studies. Dordrecht, Holland. Kluwer Academic Publishers. p. 181-208.

Donoso C. 1981. Tipos forestales de los bosques nativos de Chile. Documento de trabajo $\mathrm{N}^{\mathrm{o}}$ 38. Investigación y Desarrollo Forestal. Santiago, Chile. CONAF-FAO. 70 p.

Donoso C. 1982. Reseña ecológica de los bosques mediterráneos de Chile. Bosque 4 (2): 117-146.

Donoso C. 1993. Bosques templados de Chile y Argentina: variación, estructura y dinámica. Santiago, Chile. Editorial Universitaria. $484 \mathrm{p}$.

Eastman R. 2006. IDRISI Andes guide to GIS and image processing. Worcester, USA. Clark University. 327 p.

Ekstrand SP. 1990. Detection of moderate damage on norway spruce using Landsat TM and digital stand data. IEEE Transactions on Geoscience and Remote Sensing 28 (4): 685-692.

Entcheva Campbell PK, BN Rock, ME Martin. 2004. Detection of initial damage in Norway spruce canopies using hyperspectral airborne data. International Journal of Remote Sensing 25 (24): 5557-5583.

Gitelson AA, YJ Kaufman, R Stark, D Rundquist. 2002. Novel algorithms for remote estimation of vegetation fraction. Remote Sensing of Environment 80: 76-87.

Govender M, K Chetty, H Bulcock. 2007. A review of hyperspectral remote sensing and its application in vegetation and water resource studies. Water SA 33 (2): 145-152. 
Greslebin AG, EM Hansen, W Sutton. 2007. Phytophthora austrocedrae sp. nov., a new species associated with Austrocedrus chilensis mortality in Patagonia (Argentina). Mycological Research 111 (3): 308-316.

Greslebin AG, EM Hansen, L Winton, M Rajchenberg. 2005. Phytophthora species from declining Austrocedrus chilensis forests in Patagonia, Argentina. Mycologia 97: 218-228.

Holmgren A. 2006. Evaluación del efecto de Cinara cupressi (Buckton) (Hemiptera, Aphididae) post control químico, sobre Austrocedrus chilensis (D. Don). Tesis Ingeniero Forestal. Santiago, Chile. Facultad de Agronomía e Ingeniería Forestal, Pontificia Universidad Católica de Chile. 65 p.

Huete A, K Didan. 2004. MODIS seasonal and inter-annual responses of semiarid ecosystems to drought in the Southwest U.S.A. IEEE International 3: 1538-1541.

Hunt ER, BN Rock. 1989. Detection of changes in leaf water content using near- and middle-infrared reflectances. Remote Sensing of Environment 30: 43-54.

Ito E, S Lim, B Tith, P Pith, S Khorn, A Tani, M Kanzaki, T Kaneko, Y Okuda, M Araki. 2007. Use of ASTER optical indices to estimate spatial variation in tropical seasonal forests on the West Bank of the Mekong River, Cambodia. In Sawada H, M Araki, NA Chappell, JV LaFrankie, A Shimizu eds. Forest Environments in the Mekong River Basin. Japan, Springer. p. 232-240.

INFOR (Instituto Forestal de Chile, CL). 2008. Manejo integrado: técnica para la recuperación del crecimiento de Austrocedrus chilensis. Santiago, Chile. Corporación de Fomento de la Producción. 129 p.

Iverson L, RL Graham, EA Cook. 1989. Applications of satellite remote sensing to forested ecosystems. Landscape Ecology 3 (2): 131-143.

Kharuk VI, KJ Ranson, VV Kuz'michev, ST Im. 2003. Landsatbased analysis of insect outbreaks in southern Siberia. Canadian Journal of Remote Sensing 29(2): 286-297.

Karnieli A, YJ Kaufman, L Remer, A Wald. 2001. AFRI-aerosol free vegetation index. Remote Sensing of Environment 77: $10-21$.

Komura R, M Kubo, N Kamata, K Muramoto. 2003. Analysis of Forest Damage by Harmful Insects on Mt. Kariyasu. In SICE 2003 Annual Conference. Fukui University. Fukui, Japan. p. 437-440.

Lawrence R, M Labus. 2003. Early detection of Douglas-Fir beetle infestation with subcanopy resolution hyperspectral imagery. Western Journal of Applied Forestry 18: 202-206.

Lillesand TM, RW Kiefer, JW Chipman. 2004. Remote sensing and image interpretation. New York, USA. Wiley. 763 p.

Lowe S, M Browne, S Doudjelas, M De Poorter. 2000. 100 of the world's worst invasive alien species. A selection from the global invasive species database. Auckland: The Invasive Species Specialist Group (ISSG). 12 p.

Lucas R, A Rowlands, O Niemann, R Merton. 2004. Hyperspectral sensors and applications. In Varshney PK, MK Arora eds. Advanced Image Processing Techniques for Remotely Sensed Hyperspectral Data. Berlin, Germany. Springer. p. 11-49.

Luebert F, P Pliscoff. 2006. Sinopsis bioclimática y vegetacional de Chile. Santiago, Chile. Editorial Universitaria. 316 p.
Niemann KO, F Visintini. 2004. Assessment of potential remote sensing detection of bark beetle infested areas during green attack: a literature review. Victoria, Canada. Her Majesty the Queen in Right of Canada. 14 p.

Peña MA, SH Altmann. 2009. Use of satellite-derived hyperspectral indices to identify stress symptoms in an Austrocedrus chilensis forest infested by the aphid Cinara cupressi. International Journal of Pest Management 55 (3): 197-206.

Radeloff VC, DJ Mladenoff, MS Boyce. 1999. Detecting jack pine budworm defoliation using spectral mixture analysis -a regional measure abundance from multispectral images. Remote Sensing of Environment 69 (2): 156-169.

Schlegel FM. 1962. Hallazgo de un bosque de cipreses cordilleranos en la provincia de Aconcagua. Boletín de la Universidad de Chile 32: 43-46.

Schmithüsen J. 1960. Die Nadelhölzer in den Waldgesellschaften der südlichen Anden. Vegetatio 9: 313-327.

Rodríguez R. 2004. Monografía ciprés de la cordillera (Austrocedrus chilensis). Especie con problemas de conservación en Chile. Santiago, Chile. Endesa. 71 p.

Stimson HC, DD Breshears, SL Ustin, SC Kefauver. 2005. Spectral sensing of foliar water conditions in two co-ocurring conifer species: Pinus edulis and Juniperus monosperma. Remote Sensing of Environment 96: 108-118.

Stone C, NC Coops. 2004. Assessment and monitoring of damage from insects in Australian eucalypt forests and commercial plantations. Australian Journal of Entomology. 43: 283-292.

Treitz PM, PJ Howarth. 1999. Hyperspectral remote sensing for estimating biophysical parameters of forest ecosystems. Progress in Physical Geography 23 (3): 359-390.

UICN (Unión Internacional para la Conservación de la Naturaleza y de los Recursos Naturales). 2001. Categorías y criterios de la lista roja de la UICN: versión 3.1. Gland, Suiza y Cambridge, Reino Unido. Comisión de Supervivencia de Especies de la UICN. 33 p.

Vogelmann JE. 1990. Comparison between two vegetation indices for measuring different types of forest damage in the northeastern United States. International Journal of Remote Sensing 11 (12): 2281-2297.

Vogelmann JE, BN Rock. 1989. Use of Thematic Mapper data for the detection of forest damage caused by the pear thrips. Remote Sensing of Environment 30: 217-225.

Wulder MA, SE Franklin. 2003. Remote sensing of forest environments, introduction: the transition from theory to information. In Wulder MA, SE Franklin eds. Remote Sensing of Forest Environments: Concepts and Case Studies. Dordrecht, Holland. Kluwer Academic Publishers. p. 3-12.

Young JA, DD Morton. 2002. Modeling Landscape-Level Impacts of HWA in Shenandoah National Park. In Proceedings: Hemlock Woolly Adelgid in the Eastern United States Symposium. Agricultural Experiment Station, Rutgers University. New Brunswick, USA. p. 73-85.

Zhang X, G Yan, Q Li, H Wan, Z Guo. 2006. Evaluating the fraction of vegetation cover based on NDVI spatial scale correction model. International Journal of Remote Sensing 27 (24): 5359-5372. 